\title{
Resveratrol affects histone 3 lysine 27 methylation of vessels and blood biomarkers in DOCA salt-induced hypertension
}

\author{
Sevtap Han • Mecit Orhan Uludag • \\ Suzan Emel Usanmaz • Fatma Ayaloglu-Butun • \\ Kamil Can Akcali · Emine Demirel-Yilmaz
}

Received: 3 March 2014/ Accepted: 10 September 2014/Published online: 19 September 2014

(C) Springer Science+Business Media Dordrecht 2014

\begin{abstract}
Hypertension is a risk factor for the cardiovascular diseases. Although, several drugs are used to treat hypertension, the success of the antihypertensive therapy is limited. Resveratrol decreases blood pressure in animal models of hypertension. This study researched the mechanisms behind the effects of resveratrol on hypertension. Hypertension was induced by using the deoxycorticosterone acetate (DOCA)-induced $(15 \mathrm{mg} / \mathrm{kg}$ twice per week, subcutaneously) salt-sensitive hypertension model of Wistar rats. Hypertension caused a decrease in endotheliumdependent relaxations of the isolated thoracic aorta. Resveratrol treatment $(50 \mathrm{mg} / \mathrm{l}$ in drinking water) prevented DOCA salt-induced hypertension, but did not improve endothelial dysfunction. Plasma nitric oxide (NO), asymmetric dimethylarginine (ADMA), total antioxidant capacity (TAC) and hydrogen sulfide $\left(\mathrm{H}_{2} \mathrm{~S}\right)$ levels were not changed by DOCA salt application. However, treatment of resveratrol significantly decreased ADMA and increased TAC and $\mathrm{H}_{2} \mathrm{~S}$ levels. NO level in circulation was not significantly changed by resveratrol. DOCA salt application
\end{abstract}

S. Han - M. O. Uludag

Department of Pharmacology, Faculty of Pharmacy, Gazi

University, Etiler, 06330 Ankara, Turkey

S. E. Usanmaz · E. Demirel-Yilmaz ( $)$

Department of Medical Pharmacology, Faculty of Medicine, Ankara University, Sihhiye, 06100 Ankara, Turkey

e-mail: dyilmaz@medicine.ankara.edu.tr

F. Ayaloglu-Butun

Department of Molecular Biology and Genetics, Faculty of

Science, Bilkent University, Bilkent, 06800 Ankara, Turkey

K. C. Akcali

Department of Biophysics, Faculty of Medicine, Ankara

University, Sihhiye, 06100 Ankara, Turkey and resveratrol treatment also caused an alteration in the epigenetic modification of vessels. Staining pattern of histone 3 lysine 27 methylation (H3K27me3) in the aorta and renal artery sections was changed. These results show that preventive effect of resveratrol on DOCA salt-induced hypertension might due to its action on the production of some blood biomarkers and the epigenetic modification of vessels that would focus upon new aspect of hypertension prevention and treatment.

Keywords Hypertension - Resveratrol - Endothelium · Biomarker · Epigenetic modification

\section{Introduction}

Hypertension is an increasing health problem in societies and is characterized by elevated systemic blood pressure that causes organ damage [1]. However, the exact molecular mechanism of hypertension remains unknown. It has been suggested that increased blood pressure is probably the reason or/and result of vascular endothelial dysfunction [2]. Vascular endothelium plays an important role in the regulation of blood pressure by secreting various vasoactive substances. However, it is widely accepted that production of $\mathrm{NO}$ represents the functional status of endothelial cells. Decreased bioavailability of NO in endothelial dysfunction is the result of inhibiting NO synthesis by oxidative stress and scavenging $\mathrm{NO}$ by superoxide anion. There is accumulating evidence indicating that oxidative stress is closely related to the development of hypertension [3].

Several animal models have been developed to investigate the molecular mechanisms of hypertension and to evaluate new therapeutic approaches and drugs. Although 
there are no animal models resembling features of human hypertension, deoxycorticosterone acetate (DOCA)-salt induced hypertension remains a well-established experimental approach. Although, endothelial damage in the vascular bed plays a key role in DOCA salt-induced hypertension, the correlation of endothelial functions with possible blood biomarkers such as nitric oxide (NO), asymmetric dimethylarginine (ADMA), total antioxidant capacity (TAC) and hydrogen sulfide $\left(\mathrm{H}_{2} \mathrm{~S}\right)$ were not described in detail after long term DOCA salt application.

On the other hand, the beneficial effect of resveratrol on hypertension has been reported [4-6]. Resveratrol is a natural compound and has been found to have several pharmacological effects, including vasodilator, antiinflammatory, anti-platelet, anti-oxidant, anti-fungal, antiaging and anti-cancer properties [7]. However, the underlying mechanism of the resveratrol effects on hypertension has not been fully understood. Although, some epigenetic modifications in hypertension were determined [8], effect of resveratrol on epigenetic modifications of vessels in hypertension was not examined yet.

In the current study, to further elucidate the mechanisms behind the beneficial effect of resveratrol on hypertension; endothelium-dependent relaxation of isolated thoracic aorta, blood biomarkers related to endothelial functions (NO, ADMA, TAC, $\mathrm{H}_{2} \mathrm{~S}$ ) and epigenetic modification of vessels were investigated in the rat model of the DOCA salt-induced hypertension.

\section{Materials and methods}

Animal care and surgical procedure

All experiments were carried out in compliance with the National Institute of Health Guide for the Care and Use of Laboratory Animals. The experimental procedure was approved by the Local Ethical Committee of Animal Care and Use of Ankara University. Eight-week-old male Wistar albino rats (200-240 g) were used in this study. All rats were housed in an air-conditioned room $\left(22 \pm 1{ }^{\circ} \mathrm{C}\right)$ under a $12 \mathrm{~h}$ light/dark cycle. Hypertension was induced by using the DOCA salt hypertension model of rats. In hypertension groups, animals were anesthetized with a mixture of ketamine $(60 \mathrm{mg} / \mathrm{kg})$ and xylazine $(10 \mathrm{mg} / \mathrm{kg})$ and unilateral nephrectomy was performed. After the 1 week recovery period, DOCA was administered subcutaneously $(15 \mathrm{mg} / \mathrm{kg}$ twice per week) and $1 \% \mathrm{NaCl}$ and $0.2 \% \mathrm{KCl}$ were added to their drinking water for 12 weeks. Resveratrol $(50 \mathrm{mg} / \mathrm{l})$ was administrated in drinking water of normotensive and hypertensive animal groups. Total water intake and body weight were recorded in rats and the daily dose of resveratrol was calculated approximately $7-7.5 \mathrm{mg} / \mathrm{kg}$.
During the experimental period, all rats were allowed free access to water and food. DOCA salt and resveratrol administration did not have any effect on eating and drinking behaviors of the rats as body weights were similar in all the groups at the end of the experimental period.

Blood pressure measurements

Systolic blood pressure was measured once per week on prewarmed and restrained rats by tail-cuff plethysmography (NIBP200A, COMMAT, Turkey). At least five measurements were made for each rat and the mean values were taken.

Tissue bath procedure

After the 12 weeks experimental period, the rats were anesthetized with thiopental $(40 \mathrm{mg} / \mathrm{kg})$ and blood samples were collected. Isolated ring segments of thoracic aorta were placed in organ baths containing Krebs solution (in mM: $112 \mathrm{NaCl}, 5 \mathrm{KCl}, 11.5$ Dextrose, $25 \mathrm{NaHCO}_{3}, 0.5$ $\mathrm{MgCl}_{2}, 2.5 \mathrm{CaCl}_{2}, 1.2 \mathrm{NaH}_{2} \mathrm{PO}_{4}, \mathrm{pH}=7.4$ ) aerated with $95 \% \mathrm{O}_{2}$, and $5 \% \mathrm{CO}_{2}$ and warmed $\left(37^{\circ} \mathrm{C}\right)$. Each ring was mounted between a stainless-steel hook and connected to a force displacement transducer (FDT05, May, COMMAT, Turkey) under an initial tension of $2 \mathrm{~g}$. The tissues were allowed to equilibrate for at least $40 \mathrm{~min}$ before drug treatment. Isometric contractions were displayed and recorded with Integrated Tissue Bath System (ITBS08, May, COMMAT, Turkey), MP35 data acquisition and BSL PRO 3.7 acquisition software (BIOPAC, USA). Cumulative concentration-response curves were performed for acetylcholine during the precontraction of phenylephrine. The relaxations were expressed as a percentage of the precontraction.

\section{Biochemical examinations}

The plasma nitrite level was measured as a representation of NO production. It was measured by using the spectrophotometric method based on the Griess reaction [9]. This method was modified in our laboratories for 96-well plates. The TAC of plasma was measured by the method which was described previously [10], based on the reduction of $\mathrm{Cu}^{+2}$ to $\mathrm{Cu}^{+1}$ by the antioxidants in the plasma. Neocuproine was used as a chromogenic agent and colored complex was detected spectrophotometrically, at $455 \mathrm{~nm}$. $\mathrm{H}_{2} \mathrm{~S}$ levels of the plasma were measured spectrophotometrically, according to the previously described method based on the measurements of the absorbance of the methylene blue, which produced the chemical reaction between N,N-dimethyl-p-phenylenediamine and $\mathrm{FeCl}_{3}$, at $670 \mathrm{~nm}$ [11]. ADMA levels were measured by using 


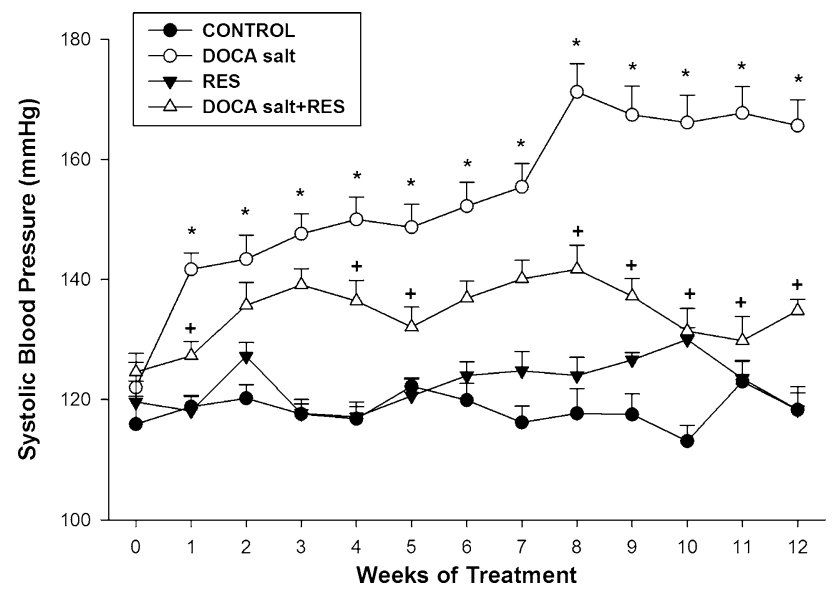

Fig. 1 Effects of chronic resveratrol treatment on the systolic blood pressures of normotensive and hypertensive rats. Resveratrol treatment significantly inhibited 12 weeks DOCA salt application-induced elevation of systolic blood pressure $(p<0.05)$. Differences from control (*) and DOCA salt $(+)$. Values are expressed as mean \pm SEM $(n=5-11)$

ELISA kits (Immunodiagnostic A.G., Germany) according to the manufacturer's instruction.

Evaluation of histon methylations

Frozen tissue samples ( $5 \mu$ thick) were fixed with freshly prepared $4 \%$ paraformaldehyde for $30 \mathrm{~min}$. After washing, slides were incubated with pre-blocking serum (bovine serum albumin $10 \%$, triton-X $0.3 \%$ ) for $1 \mathrm{~h}$ at $37{ }^{\circ} \mathrm{C}$. Primary antibodies were purchased from Cell Signaling Technology (MA, USA) and were applied (diluted 1:1000) to pre-blocking solution and kept at $+4{ }^{\circ} \mathrm{C}$ for overnight. Tissue sections were washed with $1 \times$ PBS for once and incubated with FITC-labeled anti-rabbit secondary antibodies (Sigma, St. Louis, MO, USA) at a dilution of 1/200 for $1 \mathrm{~h}$ at room temperature. After incubating in the dark for $1 \mathrm{~h}$ at room temperature, the specimens were mounted using UltraCruzTM (Santa Cruz) medium with DAPI and examined under a fluorescence microscope.

\section{Chemicals}

Acetylcholine, phenylephrine and DOCA were obtained from Sigma Chemical Co (St Louis, MO, USA). Trans-Resveratrol was obtained from Herb-Tech (China). Resveratrol was dissolved in $0.05 \% \mathrm{vol} / \mathrm{vol}$ ethanol and stored in dark bottles at $2-4{ }^{\circ} \mathrm{C}$. DOCA was dissolved in corn oil $(15 \mathrm{mg} / \mathrm{ml})$.

\section{Statistical analysis}

Values are expressed as mean \pm SEM. Statistical analyses were performed using the SigmaPlot (Systat Software Inc.,

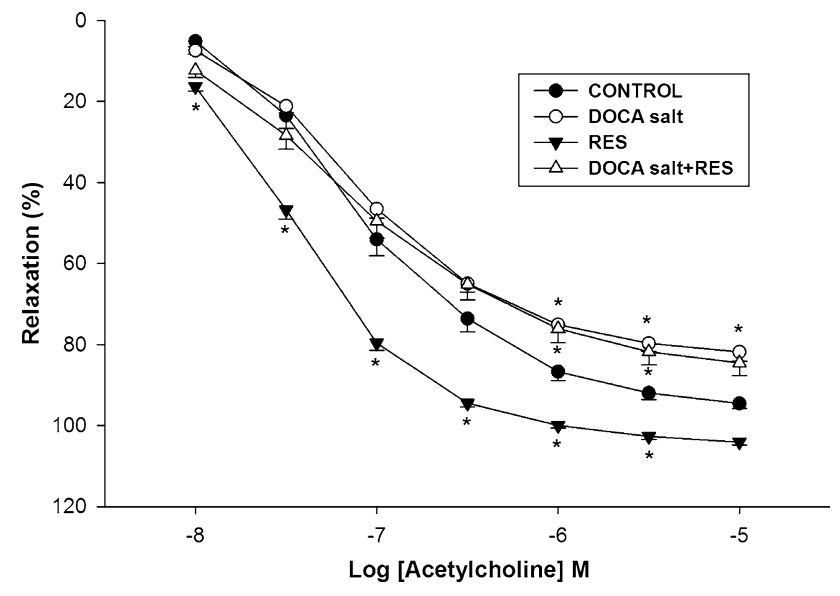

Fig. 2 Effects of chronic resveratrol treatment on the endotheliumdependent vasorelaxation of aorta isolated from normotensive and hypertensive rats. Resveratrol treatment did not alter DOCA salt application-induced decrease in the acetylcholine-induced endothelium-dependent relaxations of aorta. Endothelium-dependent relaxation of vessels was significantly enhanced by 12 weeks treatment of resveratrol in normal animals $(p<0.05)$. Differences from control (*). Values are expressed as mean $\pm \operatorname{SEM}(n=20-42)$

USA) version 11 for Windows. Differences among the groups were evaluated by Student's $t$ test. Repeated-measures of two-way ANOVA was used to test differences among the groups of blood pressure and relaxations. When the $p$ value is statistically significant, comparison procedure was applied by using the Bonferroni test. Values were considered significantly different when $p<0.05$.

\section{Results}

Basal values of systolic blood pressure were not different in all experimental groups. In the DOCA salt groups, systolic blood pressure was significantly increased at the end of 12 weeks hypertension periods, when compared to the control groups $(p<0.05)$ (Fig. 1). Elevated systolic blood pressure was significantly decreased by chronic resveratrol treatment $(p<0.05)$ (Fig. 1). Resveratrol treatment did not alter the systolic blood pressure of normal animals.

Acethylcholine-induced endothelium-dependent relaxations were obtained against phenylephrine-induced precontraction in the thoracic aorta. Acethylcholine $\left(10^{-8}\right.$ $10^{-5} \mathrm{M}$ ) caused a concentration-dependent relaxation of the vessels. Endothelium-dependent relaxations were attenuated significantly in the aorta isolated from the 12 weeks DOCA salt hypertension group $(p<0.05)$ (Fig. 2). DOCA salt hypertension-induced decrease in endothelium-dependent relaxations was not affected by resveratrol treatment in DOCA salt hypertension (Fig. 2). 
Fig. 3 Effects of chronic resveratrol treatment on the plasma Nitrite, ADMA, TAC and $\mathrm{H}_{2} \mathrm{~S}$ levels of normotensive and hypertensive rats. Plasma nitrite levels were not different in all groups. While plasma ADMA level was significantly decreased by 12 weeks resveratrol treatment, TAC and $\mathrm{H}_{2} \mathrm{~S}$ levels were significantly increased in resveratrol and DOCA salt-resveratrol groups $(p<0.05)$. Differences from control (*) and DOCA salt (+). Values are expressed as mean \pm SEM $(n=8-15)$
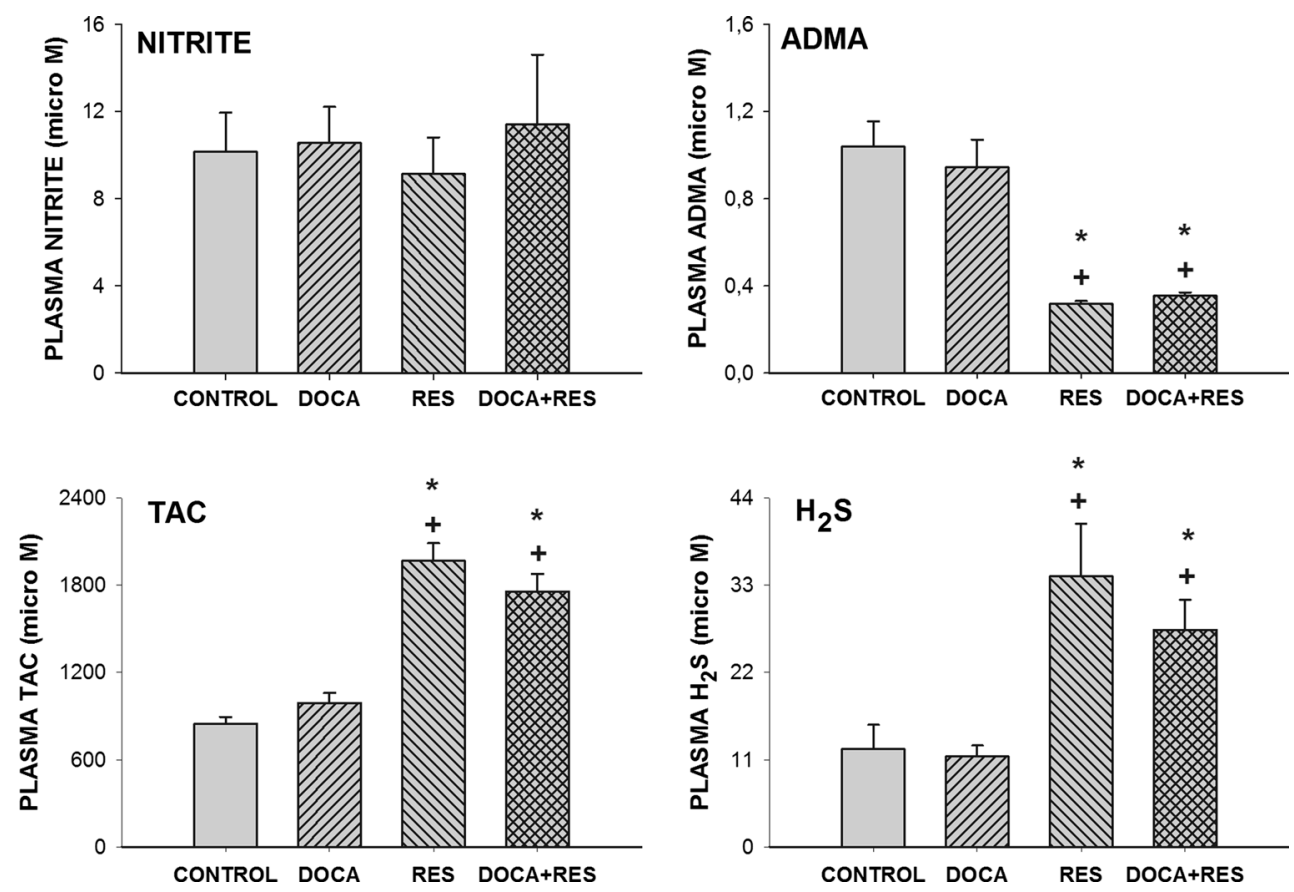

However, resveratrol treatment was significantly augmented acetylcholine-induced endothelium-dependent relaxation of vessels isolated from normal animals $(p<0.05)$ (Fig. 2).

The effect of resveratrol on the blood biomarkers related to endothelial functions was examined by measuring plasma NO, ADMA, TAC and $\mathrm{H}_{2} \mathrm{~S}$ levels. The plasma nitrite level as a representative of NO production was not significantly different in any of the groups (Fig. 3). Resveratrol treatment significantly decreased plasma ADMA levels $\left(p<0.05\right.$ ) (Fig. 3). On the other hand, TAC and $\mathrm{H}_{2} \mathrm{~S}$ levels were significantly increased by resveratrol treatment $(p<0.05)$ (Fig. 3).

Epigenetic modifications were observed in the thoracic aorta and renal artery. The expression of $\mathrm{H} 3 \mathrm{~K} 4 \mathrm{me} 1$, H3K4me3, H3K9me2, H3K9me3, H3K27me2, H3K27me3, H3K36me2 and H3K36me3 were examined in all groups but, we observed only difference in the staining of H3K27me3 (Fig. 4). We have examined the expression of $\mathrm{H} 3 \mathrm{~K} 27 \mathrm{me} 3$ both in aorta and renal artery. Positive staining of $\mathrm{H} 3 \mathrm{~K} 27 \mathrm{me} 3$ was observed in the exterior region of the aorta isolated from DOCA salt hypertensive rats (Fig. 4A upper panel). Interestingly, expression of H3K27me3 pervaded the whole aorta tissue with resveratrol treatment in hypertensive rats (Fig. 4E upper panel). While there was a slight staining in the exterior region in control rats (Fig. 4C upper panel), H3K27me3 expression became more diffuse within all layers of aorta with resveratrol treatment (Fig. 4G upper panel). In the renal artery, there was a slight staining in the exterior region in the hypertensive rats (Fig. 4A lower panel), while the control group positive staining was present in all layers of renal artery (Fig. 4C lower panel). Interestingly the expression was diminished in the renal artery when resveratrol was given to normal rats (Fig. 4G lower panel), but not in the hypertensive animals (Fig. 4E lower panel). Our results showed that resveratrol treatment not only increased the positive staining in hypertensive rats, but also excessively reduced the positive staining in the renal artery isolated from normotensive animals.

\section{Discussion}

The results of this study demonstrate that the actions of resveratrol on epigenetic modifications of a vessel wall and the production of some blood biomarkers might be responsible for its beneficial effect on DOCA salt-induced hypertension.

Sustained elevation of systemic blood pressure is the main component of hypertension but, its pathophysiology is not fully understood and therefore clinical therapy is still limited in this chronic disease [1]. Resveratrol is a natural polyphenol present in plants and various human foods. It has been demonstrated that resveratrol has several pharmacological effects, including anti-oxidant, anti-atherogenic, anti-inflammatory, anti-platelet, anti-fungal, antiaging and anti-cancer properties [8]. In the cardiovascular system, resveratrol is able to decrease contractions in the cardiac and vascular smooth muscle [12-14]. In addition, the beneficial effect of resveratrol on hypertension has been 


\section{AORTA}
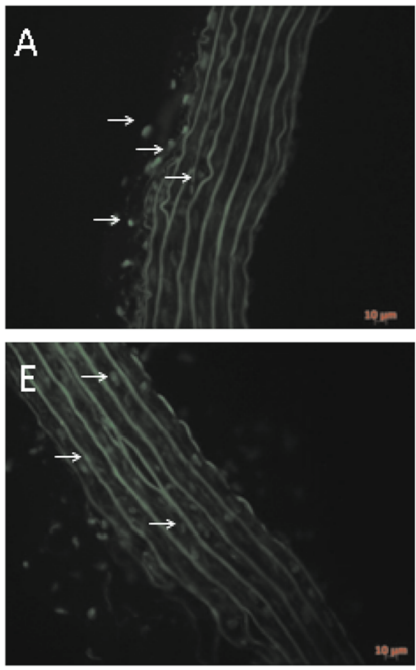

$\mathrm{H} 3 \mathrm{~K} 27 \mathrm{~m} 3$

RENAL ARTERY
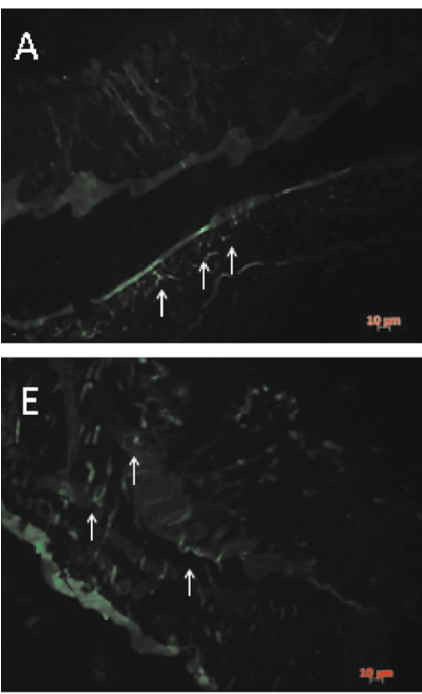

$\mathrm{H} 3 \mathrm{~K} 27 \mathrm{~m} 3$
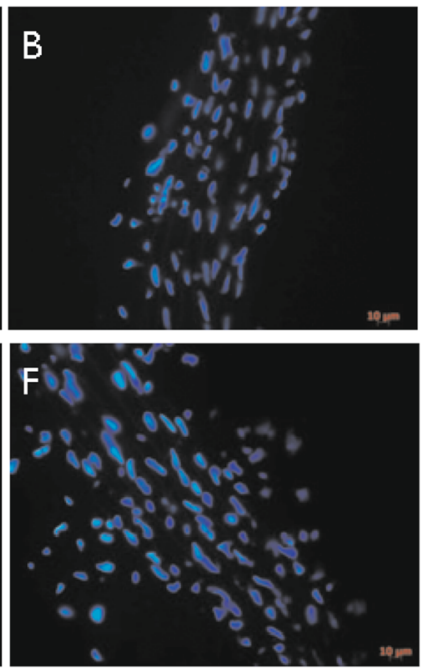

DAPI
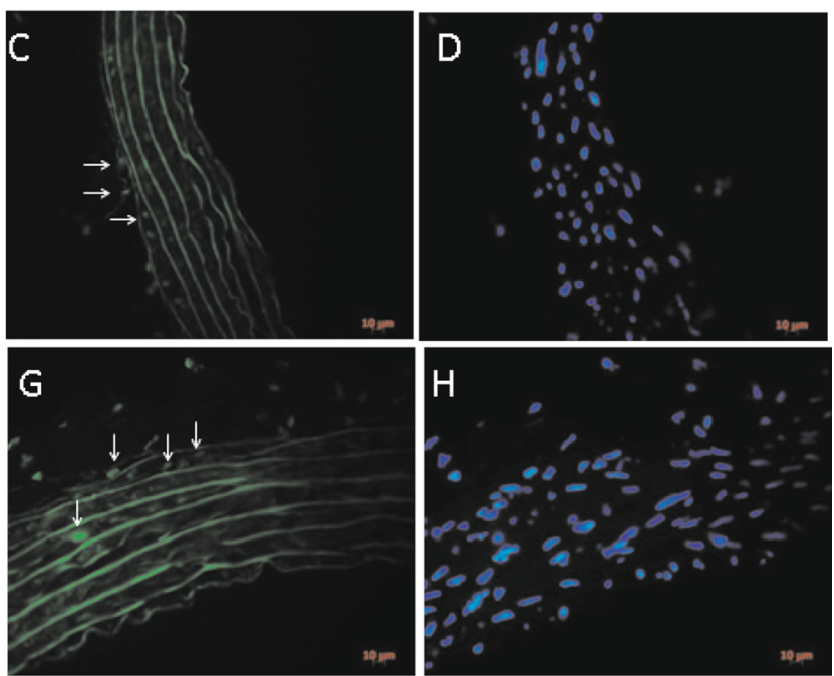

$H 3 K 27 \mathrm{~m} 3$

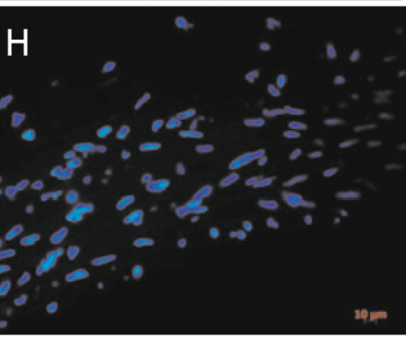

DAPI
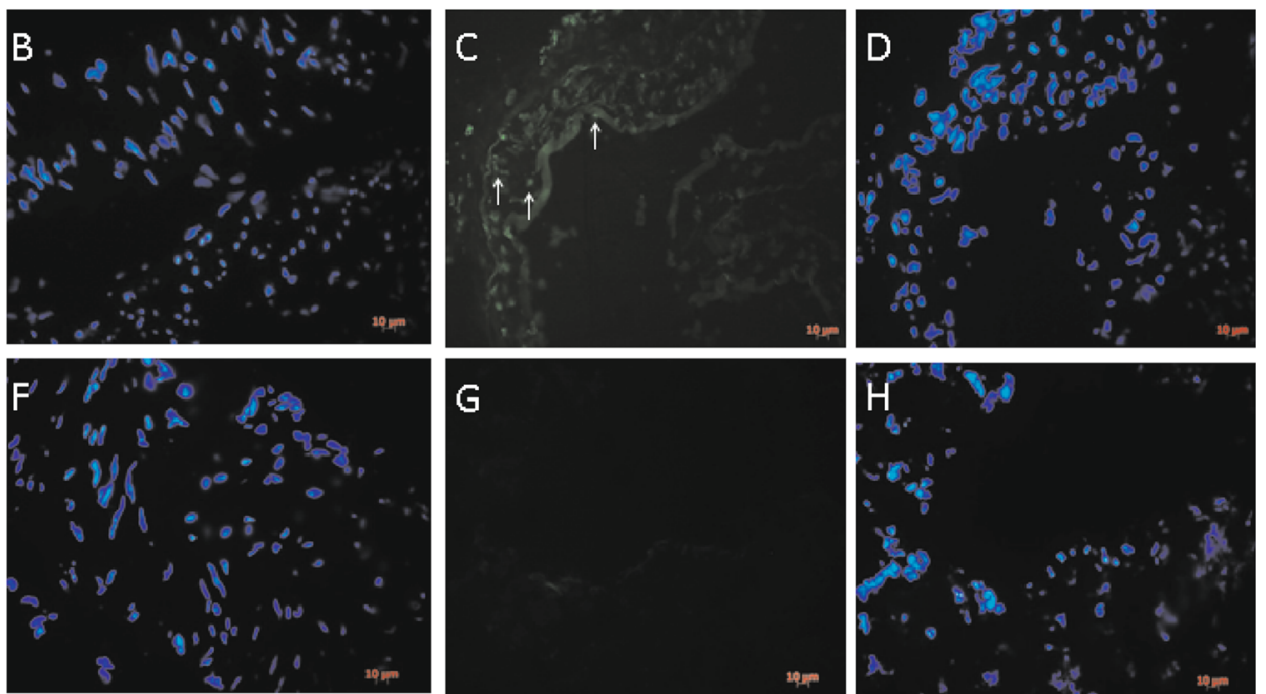

DAPI

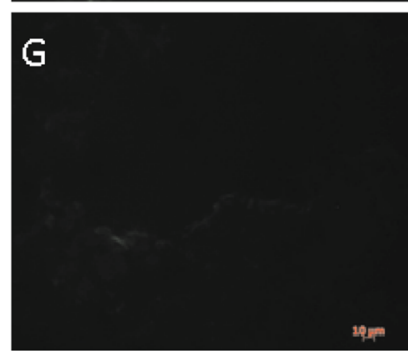

$\mathrm{H} 3 \mathrm{~K} 27 \mathrm{~m} 3$

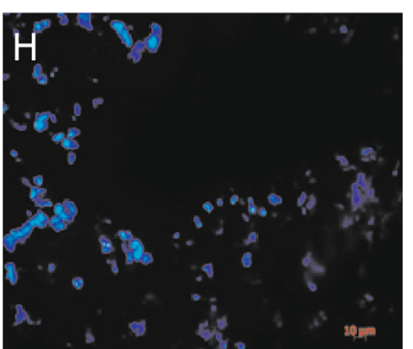

DAPI

resveratrol increased the positive staining in hypertensive rats but it reduced the positive staining in renal artery isolated from normotensive animals. A DOCA-salt group, C Control group, E DOCA salt-resveratrol group, G Resveratrol group. Nuclei are stained by DAPI (B, D, F, H). Arrows depict some of cells with positive staining

thoracic aorta isolated from the DOCA salt group similar to the previous report [15]. The beneficial action of resveratrol on relaxations of vessels isolated from spontaneously hypertensive and Dahl-salt sensitive rats has been examined [5-7]. However, the effect of resveratrol on the DOCA saltinduced decrease in the endothelium-dependent relaxations was not determined. In the present study, resveratrol did not affect DOCA salt-induced failure in endothelium-dependent relaxation of aorta. However, relaxations of vessels isolated

the endothelial cell. In our study, acetylcholine-indu endothelium-dependent relaxations were decreased in the 
from normotensive animals were augmented by resveratrol. A similar effect of resveratrol on vessels isolated from normotensive animals had been examined in Wistar rats [16] but not in Sprague-Dawley rats [5]. Thus, these results represented that the effect of resveratrol on the endotheliumdependent relaxation was related to animal species and hypertension models.

On the other hand, NO could be derived from other cells in pathological conditions. It has been reported that inflammation plays a role in the pathophysiology of the hypertension and would stimulate NO production from immune cells [17]. Unchanged or reduced plasma levels of NO had been demonstrated in human and animal hypertensions $[18,19]$. In our study, the plasma NO level was not affected by application of DOCA salt. Decreased level of endothelium-derived NO production might be balanced by NO secreted from immune cell. Thus, it was hypothesized that inflammatory reaction might be responsible for the unchanged level of NO in systemic circulation. In addition, resveratrol did not alter the plasma NO level in the current study. The preventive effect of resveratrol on hypertension-induced decreases in the NO level had been observed [6, 20,21]. Because of the use of different experimental models and timing in hypertension, it is hard to compare that data and understand the nature of the NO level as a biomarker. Further studies are needed for the time course of blood NO levels in hypertension.

ADMA is an endogenous competitive inhibitor of NO synthase and increased level of ADMA causes NO reduction in different types of hypertension [16, 19, 22]. However, the blood ADMA level was not determined in DOCA salt-induced hypertension of rats. In our study, the ADMA level was not changed in DOCA salt-induced hypertension. In addition, this study showed for the first time that resveratrol caused a reduction of the blood ADMA level in hypertensive and normotensive animals. Thus, it is suggested that the action of resveratrol on an ADMA metabolism may be another important mechanism for the beneficial effect on cardiovascular functions.

Elevated reactive oxygen species-induced oxidative stress had been reported in human and different animal models of hypertension [23-25]. Oxidative stress causes the reduction of NO bioavailability due to removing NO by a super oxide radical and decreasing NO synthesis/release from endothelium. In the current study, plasma TAC levels were not affected in DOCA salt hypertension. However, resveratrol increased plasma TAC levels of normotensive and hypertensive animals. The antioxidant properties of resveratrol were observed previously by other investigators using different hypertension models and durations [6, 26].

$\mathrm{H}_{2} \mathrm{~S}$ is a new gas mediator and has ben shown to have a vital role in various physiological functions including cardiovascular modulation. It had been reported that $\mathrm{H}_{2} \mathrm{~S}$ has a regulatory role in different types of hypertension [27]. The $\mathrm{H}_{2} \mathrm{~S}$ level in plasma was not determined in the DOCA salt-induced hypertension. In the present study, DOCA salt application did not significantly alter the plasma $\mathrm{H}_{2} \mathrm{~S}$ level but, resveratrol treatment increased the plasma level of $\mathrm{H}_{2} \mathrm{~S}$ in normotensive and hypertensive animals. To our knowledge, effect of resveratrol on the plasma level of $\mathrm{H}_{2} \mathrm{~S}$ has not been shown before and increased level of $\mathrm{H}_{2} \mathrm{~S}$ might be responsible for the beneficial effect of resveratrol on hypertension.

DOCA salt-induced hypertension in rats is the commonly used model of hypertension. However, different dose and duration of DOCA salt application had been used in those studies. In our study, moderate dose and long term application were chosen. Although, DOCA salt treatment affected blood pressure and endothelium-dependent relaxations in the present study, blood levels of NO, ADMA, TAC and $\mathrm{H}_{2} \mathrm{~S}$ were not changed. Several factors play roles in metabolism of blood biomarkers. Dose or duration of DOCA salt application probably may not have ability to affect them.

On the other hand, both hypertensive and normotensive animals were affected by resveratrol treatment. In the normotensive animals, resveratrol changed endothelium-dependent relaxations of vessels and blood level of ADMA, TAC and $\mathrm{H}_{2} \mathrm{~S}$. Wide ranges of pharmacological effects have been observed by resveratrol in vitro and in vivo studies [7, 12-14]. It is posited that metabolizing enzymes of biomarkers might be affected by resveratrol in vivo condition. However, further studies are needed to better understand molecular mechanisms of this interaction.

Beside the other effects, resveratrol causes epigenetic modifications due to protein sirtuins (SIRT1,3,4,7)-mediated mechanisms [28]. Epigenetic mechanisms regulate gene expression in somatic development and life-long health and disease. In the cell nucleus, nucleosome is composed of DNA wrapped around the histone H2A, H2B, $\mathrm{H} 3$ and $\mathrm{H} 4$ proteins. Epigenetic regulation comprises several molecular processes, including DNA methylations, histone modifications (acetylation, methylation, phosphorylation, ubiquitination, citrullination and sumoylation), nucleosome positioning, transcription control with DNA binding proteins and non-coding RNAs, and translation control with microRNAs and RNA binding proteins [29]. Influence of resveratrol on SIRT enzymes affects histone acetylation [28]. However, acetylation have been shown to act on DNA and histone methylations as well [30, 31]. Although, hypertension-induced epigenetic modifications had been reported [4], effect of resveratrol on hypertension-induced histone methylations was not investigated. In the current study, it was firstly observed that DOCA salt 
application and resveratrol treatment altered the H3K27me 3 staining of aorta and renal artery. It is evident that $\mathrm{H} 3 \mathrm{~K} 27 \mathrm{me} 3$ staining pervaded the all layers of aorta both in hypertensive and normotensive animals upon resveratrol administration. On the other hand, in the renal artery, there was a different response to resveratrol. It was also clearly shown that resveratrol administration resulted in the spreading of $\mathrm{H} 3 \mathrm{~K} 27 \mathrm{me} 3$ expression to all layers of renal artery in the hypertensive rats but completely diminished its expression in normotensive animals. $\mathrm{H} 3 \mathrm{~K} 27 \mathrm{me} 3$ is associated with the gene repression and silenced chromatin and important epigenetic reprogramming of somatic and germ cells [32]. Thus, it could be posited that preventive action of resveratrol on DOCA saltinduced hypertension might be associated to its effect on epigenetic modifications. However, further studies are needed to identify the amount of epigenetic alteration and exact mechanism that is responsible for alteration of H3K27me3 methylation in DOCA salt-induced hypertension and resveratrol treatment.

All these results indicate that the beneficial effect of resveratrol on DOCA salt-induced hypertension might be related to its actions on blood ADMA, TAC and $\mathrm{H}_{2} \mathrm{~S}$ levels and epigenetic modifications of vessels which could affect cardiovascular functions. Determining the molecular mechanism of resveratrol actions in hypertension may lead to the development of novel strategies to prevent or treat hypertension.

Acknowledgments This study was supported by a research Grants from the Novartis Research Award. We are grateful to Maggie Li for the editing of the English.

\section{References}

1. Coffman TM (2011) Under pressure: the search for the essential mechanisms of hypertension. Nat Med 17(11):1402-1409

2. Tang EH, Vanhoutte PM (2010) Endothelial dysfunction: a strategic target in the treatment of hypertension? Pflugers Arch 459(6):995-1004

3. Schulz E, Gori T, Münzel T (2011) Oxidative stress and endothelial dysfunction in hypertension. Hypertens Res 34(6): 665-673

4. Rush JW, Quadrilatero J, Levy AS, Ford RJ (2007) Chronic resveratrol enhances endothelium-dependent relaxation but does not alter eNOS levels in aorta of spontaneously hypertensive rats. Exp Biol Med 232(6):814-822

5. Bhatt SR, Lokhandwala MF, Banday AA (2011) Resveratrol prevents endothelial nitric oxide synthase uncoupling and attenuates development of hypertension in spontaneously hypertensive rats. Eur J Pharmacol 667(1-3):258-264

6. Rimbaud S, Ruiz M, Piquereau J, Mateo P, Fortin D, Veksler V et al (2011) Resveratrol improves survival, hemodynamics and energetics in a rat model of hypertension leading to heart failure. PLOS ONE 6(10):e26391

7. Petrovski G, Gurusamy N, Das DK (2011) Resveratrol in cardiovascular health and disease. Ann NY Acad Sci 1215:22-33
8. Cowley AW Jr, Nadeau JH, Baccarelli A, Berecek K, Fornage M, Gibbons GH et al (2012) Report of the national heart, lung, and blood institute working group on epigenetics and hypertension. Hypertension 59(5):899-905

9. Navarro-Gonzalvez J, Garcia-Benayas C, Arenas J (1998) Semiautomated measurement of nitrate in biological fluids. Clin Chem 44(3):679-681

10. Usanmaz SE, Demirel Yilmaz E (2008) A microplate based spectrophotometric method for the determination of the total antioxidant capacity of human plasma: modified cupric reducing ability assay. Fundam Clin Pharmacol 22(67-67 Suppl):2

11. Zhang H, Moochhala SM, Bhatia M (2008) Endogenous hydrogen sulfide regulates inflammatory response by activating the ERK pathway in polymicrobial sepsis. J Immunol 181: 4320-4331

12. Buluc M, Demirel-Yllmaz E (2002) Possible mechanism for depression of smooth muscle tone by resveratrol. In: Varro A, Vegh A (eds) Advances in Recent Cardiovascular Research. Monduzzi Editore, Bologna, pp 55-59

13. Buluc M, Demirel-Yilmaz E (2006) Resveratrol decreases calcium sensitivity of vascular smooth muscle and enhances cytosolic calcium increase in endothelium. Vascul Pharmacol 44(4): 231-237

14. Buluc M, Ayaz M, Turan B, Demirel-Yilmaz E (2007) Resveratrol-induced depression of the mechanical and electrical activities of the rat heart is reversed by glyburide: evidence for possible K(ATP) channels activation. Arch Pharm Res 30(5): 603-607

15. Lind L, Granstam SO, Millgård J (2000) Endothelium-dependent vasodilation in hypertension: a review. Blood Press 9(1):4-15

16. Soylemez S, Sepici A, Akar F (2009) Resveratrol supplementation gender independently improves endothelial reactivity and suppresses superoxide production in healthy rats. Cardiovasc Drugs Ther 23(6):449-458

17. Montecucco F, Pende A, Quercioli A, Mach F (2011) Inflammation in the pathophysiology of essential hypertension. J Nephrol 24(1):23-34

18. Landmesser U, Dikalov S, Price SR, McCann L, Fukai T, Holland SM et al (2003) Oxidation of tetrahydrobiopterin leads to uncoupling of endothelial cell nitric oxide synthase in hypertension. J Clin Invest 111(8):1201-1209

19. Martin C, Cameron J, McGrath B (2008) Mechanical and circulating biomarkers in isolated clinic hypertension. Clin Exp Pharmacol Physiol 35(4):402-408

20. Liu Z, Song Y, Zhang X, Liu Z, Zhang W, Mao W, Wang W, Cui W, Zhang X, Jia X, Li N, Han C, Liu C (2005) Effects of transresveratrol on hypertension-induced cardiac hypertrophy using the partially nephrectomized rat model. Clin Exp Pharmacol Physiol 32(12): 1049-1054

21. Chander V, Chopra K (2006) Possible role of nitric oxide in the protective effect of resveratrol in 5/6th nephrectomized rats. J Surg Res 133(2):129-135

22. Li J, Zhou Z, Jiang DJ, Li D, Tan B, Liu H et al (2007) Reduction of NO- and EDHF-mediated vasodilatation in hypertension: role of asymmetric dimethylarginine. Clin Exp Hypertens 29(7): 489-501

23. Borde P, Mohan M, Kasture S (2011) Effect of myricetin on deoxycorticosterone acetate (DOCA)-salt-hypertensive rats. Nat Prod Res 25(16):1549-1559

24. Castro MM, Rizzi E, Ceron CS, Guimaraes DA, Rodrigues GJ, Bendhack LM, Gerlach RF, Tanus-Santos JE (2012) Doxycycline ameliorates $2 \mathrm{~K}-1 \mathrm{C}$ hypertension-induced vascular dysfunction in rats by attenuating oxidative stress and improving nitric oxide bioavailability. Nitric Oxide 26(3):162-168

25. Gómez-Guzmán M, Jiménez R, Sánchez M, Zarzuelo MJ, Galindo P, Quintela AM et al (2012) Epicatechin lowers blood 
pressure, restores endothelial function, and decreases oxidative stress and endothelin-1 and NADPH oxidase activity in DOCAsalt hypertension. Free Radic Biol Med 52(1):70-79

26. Mizutani K, Ikeda K, Nishikata T, Yamori Y (2000) Phytoestrogens attenuate oxidative DNA damage in vascular smooth muscle cells from stroke-prone spontaneously hypertensive rats. J Hypertens 18(12):1833-1840

27. Yan H, Du J, Tang C (2004) The possible role of hydrogen sulfide on the pathogenesis of spontaneous hypertension in rats. Biochem Biophys Res Commun 313(1):22-27

28. Houtkooper RH, Pirinen E, Auwerx J (2012) Sirtuins as regulators of metabolism and healthspan. Nat Rev Mol Cell Biol 13(4):225-238

29. Arrowsmith CH, Bountra C, Fish PV, Lee K, Schapira M (2012) Epigenetic protein families: a new frontier for drug discovery. Nat Rev Drug Discov 11(5):384-400
30. Wu LP, Wang X, Li L, Zhao Y, Lu S, Yu Y et al (2008) Histone deacetylase inhibitor depsipeptide activates silenced genes through decreasing both $\mathrm{CpG}$ and $\mathrm{H} 3 \mathrm{~K} 9$ methylation on the promoter. Mol Cell Biol 28(10):3219-3235

31. Qian W, Miki D, Zhang H, Liu Y, Zhang X, Tang K et al (2012) A histone acetyltransferase regulates active DNA demethylation in Arabidopsis. Science 336(6087):1445-1448

32. Justin N, De Marco V, Aasland R, Gamblin SJ (2010) Reading, writing and editing methylated lysines on histone tails: new insights from recent structural studies. Curr Opin Struct Biol 20(6):730-738 